\title{
Effect of Nanoindentation Rate on Plastic Deformation in Cu Thin Films
}

\author{
Dariusz Chocyk ${ }^{1 *}$, Tomasz Zientarski ${ }^{2}$ \\ 1 Department of Applied Physics, Lublin University of Technology, ul. Nadbystrzycka 38, 20-618 Lublin, Poland \\ 2 Department of Computer Science, Lublin University of Technology, ul. Nadbystrzycka 36B, 20-618 Lublin, Poland \\ * Corresponding author's e-mail: d.chocyk@pollub.pl
}

\begin{abstract}
The paper investigates the nanoindentation process with different rates in the $\mathrm{Cu}(001)$ of FCC system. The indentation process was done using molecular dynamics simulation based on the embedded atom method theory and Morse potential. Simulation process of indentation used a rigid spherical indenter with the diamond structure. To structure characterization we applied the adaptive common neighbour and the dislocation extraction analysis. It was found that the range of the linear change of the indentation force depends on the rate of response of the system. The initial range of the linear dependence of stress evolution also depends on the rate of indentation. Moreover, the average total normal stress in the system is only compressive. After linear changes, we observe oscillating changes in stress evolution. During indentation, for the range of linear changes of stress, dislocations aggregated only around the indenter surface. The creation of dislocations is directly connected with the structural changes. The structure analysis revealed the formation of HCP and BCC structure in the $\mathrm{Cu}(001)$ of FCC systems and a correlation with the creation of dislocations.
\end{abstract}

Keywords: nanoindentation, stress distribution, dislocation, common neighbor analysis, molecular dynamics simulation, $\mathrm{Cu}$, thin film.

\section{INTRODUCTION}

Metallic thin films layers are commonly used in many advanced technologies including, for example, nanoelectronics [1, 2], photovoltaic [3, 4], and energy storing $[5,6]$. In each application, the mechanical properties of thin layers are important for the operation and life of the devices. Moreover, the mechanical properties of thin films are affected by the type of materials $[7,8,9]$, their roughness $[9 \div 11]$, structure and structural defects $[12 \div 17]$.

A commonly used method for testing the properties of thin layers of materials is nanoindentation. The indentation allows you to study the mechanical reaction of the material to the load of the indenter. This reaction is directly related to the structural properties of the analysed layer and is the result of changes caused by the action of the indenter. Moreover, nanoindentation gives an insight into the beginning of plastic deformation. During loading, the material under the indenter tip initially deforms elastically. Transitions to the plastic regime are often associated with a decrease in the force-depth curve, which may be associated with the nucleation of dislocations in the system $[19 \div 22]$.

The vast majority of simulation studies on the influence of indentation parameters on substrate deformation use molecular dynamics simulations for this purpose. Often, molecular dynamics (MD) simulation was applied to track the deformation dynamics, identify the defects structure and reveal the deformation mechanism in nanoscale polycrystals materials $[15,21 \div 29]$. For example, Kelchner et al. using molecular dynamics simulation found that the plastic yield may be connected with the homogeneous nucleation of dislocation loops in pure $\mathrm{Au}$. This result was confirmed by

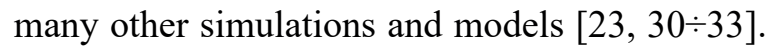
Some of these simulations give a some look into the process of homogeneous dislocation during nucleation process, suggesting existence some 
undetectable dislocation activity [33, 34]. This activity may precedes the first drop. Authors suggest that it is a heterogeneous process connected with activation of dislocation. This process is probably too subtle to detect experimentally. A new look at this phenomenon resulted in the use of an electron microscope to observe the indentation process. Minor and co-workers $[35,36]$ have pioneered the development of a hybrid technique incorporating a nanoindenter into a transmission electron microscope. They observed the initiation of dislocation activity and the interaction of the dislocation structure with grain boundaries. They showed that the first dislocation activity corresponded closely with a significant discontinuity in the force-depth curve. Moreover, some works shown that the first drop in force-depth curve is thermally activated $[37,38]$. Typically, the leads of the indentation process at higher temperatures allows the reduction of the load required to initiate yield. This suggest that deformation mechanism is a thermally activated and that the first drop in force-depth curves may be associated with a heterogeneous process $[36,39]$.

In this work, we study correlation between structure and evolution of dislocation in $\mathrm{Cu}$ systems during nanoindentation with different rates. To investigate the structure in the systems, the dislocation extraction analysis (DXA) and an adaptive version of the common neighbour analysis (CNA) methods were applied [40, 41]. The Basinski, Duesberry, and Taylor (BDT) atomic stress definition was used to determine stress in simulated systems.

\section{SIMULATION METHOD}

In order to perform the MD simulation of the nanoindentation process of $\mathrm{Cu}$ films we use the fifth-order predictor-corrector method implemented in our own original program. The indentation process of these systems are evaluated by using MD simulation based on the embedded atom method (EAM) and Morse potential. The EAM is suitable for studying structure, defects and grain boundaries in metallic systems and processes [28]. The interactions between copper are described by EAM potential [42], where the total energy $E$ is calculated according the following expression:

$$
E=\sum_{i} F_{i}\left(\rho_{i}\right)+\frac{1}{2} \sum_{i, j(i \neq j)} \phi_{i j}\left(r_{i j}\right), \rho_{i}=\sum_{j} f\left(r_{i j}\right)_{(1)}
$$

where $F_{i}\left(\rho_{i}\right)$ represents the embedding energy and $f\left(r_{i j}\right)$ is the electron density function. The two-body potential between atoms $i$ and $j$ separated by distance $r_{i j}$ is represented by $\phi_{i j}$ and $\rho_{i}$ is the local electron density at site $i$. The electron density $\left(\rho_{i}\right)$ at site $i$ derived from all atoms in system. While, the interaction carbon-carbon and coppercarbon atoms in these studies uses Morse's potential. The Morse potential energy function can be described as:

$U=\sum_{i, j(i \neq j)} D_{i j}\left[e^{-2 \alpha_{i j}\left(r_{i j}-r_{0}\right)}-2 e^{-\alpha_{i j}\left(r_{i j}-r_{0}\right)}\right]_{(2)}$

where $D_{i j}$ denotes the cohesion energy, $\alpha_{i j}$ denotes a constant parameter taken for the bulk modulus of the material, $r_{i j}$ is the distance between atoms, and $r_{0}$ is equal to the distance at equilibrium. In our calculations, the following Morse potential parameters were used: $D_{C-C}=2.420 \mathrm{eV}, D_{C-C u}=0.100$ $\mathrm{eV}, \alpha_{C-C}=0.2555 \mathrm{~nm}^{-1}, \alpha_{C-C_{u}}=0.1700 \mathrm{~nm}^{-1}, r_{0, C-C}$ $=0.2522 \mathrm{~nm}$, and $r_{0, C-C u}=0.2200 \mathrm{~nm}[39,40,41]$.

The schematic model of the indentation process is presented in Figure 1. The indented layer of metal in model is divided into an unmovable substrate and movable layers called thermal. The substrate was six monolayers thick. Whereas, thermal layer had a thickness of 30 monolayers (about $10 \mathrm{~nm}$ ). In the substrate the atoms cannot change their position. Whereas, the atoms in the thermal layers can move during simulation process. Both layers have a FCC regular crystal with bulk crystal lattice constants equal to $a_{C u}=$ $0.362 \mathrm{~nm}$ and have (001) plane parallel to surface of sample. The simulation box is a cuboid with the size of $14.84 \times 14.84 \times 15 \mathrm{~nm}$ along the $x, y, z$ directions. In all simulations, the periodic boundary conditions were used in the $x$ and $y$ directions. Whereas, non-periodic boundary conditions is imposed in the $\mathrm{z}$ direction. To avoid escaping atoms from the simulation box, a reflecting wall was placed at top of system. Before the indentation process, the structure of the $\mathrm{Cu}$ layers was optimized using the conjugate gradient algorithm and the Fletcher-Reeves formula. Next, the system was shortly relaxed for $5 p s$ by periodical velocity rescaling. The temperature of the system was kept constant and equal to $300 \mathrm{~K}$. The simulation time step was $\Delta t=5 \mathrm{fs}$.

Due to the hardness of the diamond indenter is much higher compared to $\mathrm{Cu}$ film, so the indenter was treated as rigid body and is started at $0.2 \mathrm{~nm}$ above the sample's surface. In our simulation we used the spherical indenter $(2.8 \mathrm{~nm}$ in 


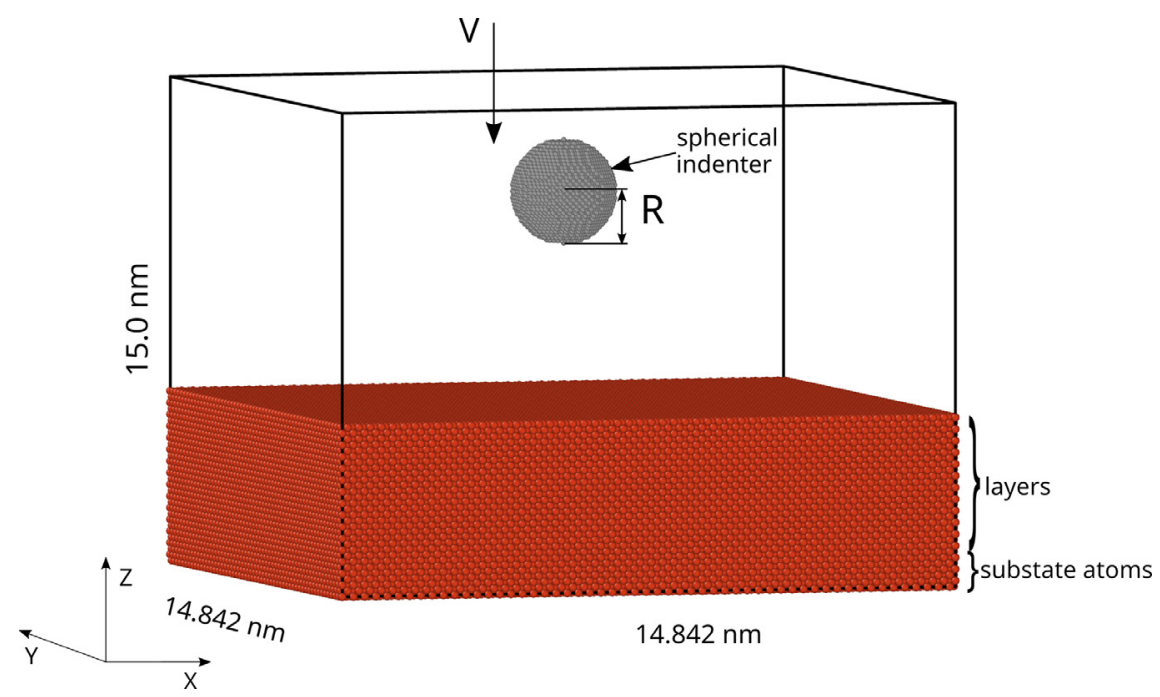

Fig. 1. Schematic model for molecular dynamic simulation

diameter). The spherical indenter with a diameter of $2.8 \mathrm{~nm}$ was used in the study. The experimentally used indenters end with a sphere. From a physical point of view, it makes no sense to indent deeper than half the diameter of the spherical indenter. Therefore, the maximum indentation depth was set to $1.4 \mathrm{~nm}$. In the literature, nanoindentation speeds are found in the range from 1 $\mathrm{m} / \mathrm{s}$ to $200 \mathrm{~m} / \mathrm{s}$. As demonstrated in [46], velocities of $100 \mathrm{~m} / \mathrm{s}$ and higher suppresses dislocation nucleation almost entirely become useless in the study of plastic effects with simulation methods. However, in the range of $10-50 \mathrm{~m} / \mathrm{s}$, the length of the dislocations shows a maximum for $20 \mathrm{~m} / \mathrm{s}$. Therefore, the indentation speeds in the range of 20 to $80 \mathrm{~m} / \mathrm{s}$ is used in our simulations.

For analyze and visualization of the structure during and post indentation process, open visualization tool (OVITO) were adopted [40]. The atomic stress (BDT) in systems was calculated using the following expression [47]:

$$
s_{i}=\frac{1}{V_{i}}\left[m_{i} \vec{v}_{i} \otimes \vec{v}_{i}+\frac{1}{2} \sum_{j \neq i}^{N} \vec{r}_{i j} \otimes \vec{f}_{i j}\right]
$$

where $m_{i}, \vec{v}_{i}$, and $V_{i}$ represents the mass, velocity, and the volume of the atom $i$, respectively. The vector $\vec{r}_{i j}=\vec{r}_{i}-\vec{r}_{j}$ denotes distance vector of atoms $i$ and $j$, and $\vec{f}_{i j}$ is the interatomic force. The total number atoms in the domain is marked by $N$ and $\otimes$ represents the tensor product of two vectors. The stress tensor elements are used to calculate stresses in the $x, y$ and $z$ directions. The average normal stress, $s_{N}$, is calculated as
$s_{N}=\left(s_{x x}+s_{y y}+s_{z z}\right) / 3$, where $s_{x x}, s_{y y}, s_{z z}$ are stress components of the stress tensor $s$.

\section{RESULTS AND DISCUSSION}

The indentation force as a function of depth for indentation rates in the range of $20 \mathrm{~m} / \mathrm{s}$ to $80 \mathrm{~m} / \mathrm{s}$, is shown in Figure 2. For all presented curves, one can observe a linear dependence on the indentation depth at the beginning processes. The linear relationship of the initial stage of indentation indicates the response of the sample is elastic. The slope of the linear part is almost the same for all the rates. The constant of the slope at the elastic stage suggests that the elastic modulus does not changes with the change of indentation rates. The maximum value of the force-depth curve increases with the growth of the indentation rate. Moreover, we observed a characteristic displacement force maximum toward to greater depth with the increase of the indentation rate.

In paper [21], in molecular dynamics simulation of nanoindentation on crystal copper, a different of force-depth evolution was also observed at the early stage of indentation after the elastic range. This kind of behavior was predicted in the analytical model taking into account nucleation, multiplication, annihilation and immobilization of dislocation around the indenter contact surface [22]. The change in the force development trend which occurs during nanoindentation is commonly associated with the start of plastic deformation processes such as dislocations and nucleation. A low indentation rate promotes more homogenous 


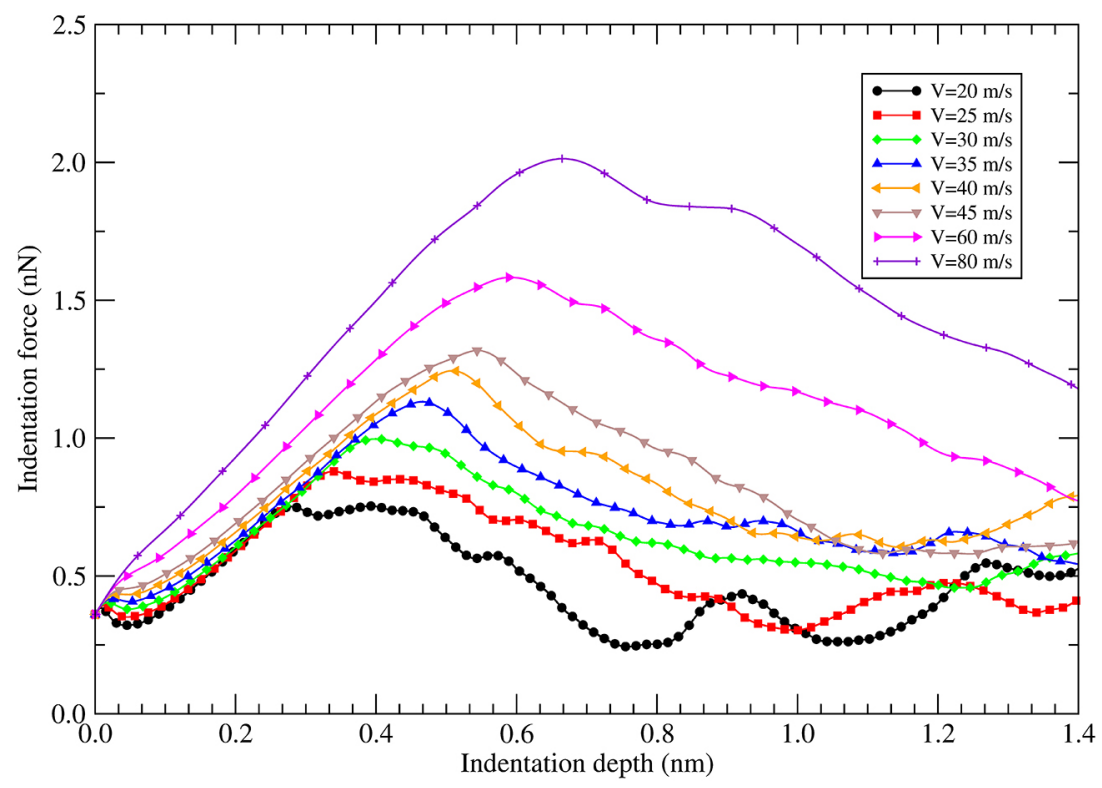

Fig. 2. Indentation force as a function of indentation depth for different rates of indentation. The indentation rate varies from $20 \mathrm{~m} / \mathrm{s}$ to $80 \mathrm{~m} / \mathrm{s}$

nucleation and propagation of lattice dislocations in the nanotwinned single crystalline than those of higher indentation rates [19].

On the one hand, at higher indentation rate, there is not enough time to relax the local stress. On the other, the higher indentation rates provide a higher energy and produce a higher density of dislocation, which is often observed experimentally [48]. The indenter immersed in the tested material supplies energy to the crystal lattice. The amount of energy supplied depends on the rate of indentation. This energy can affect nanoscale plasticity mechanisms and thermal softening processes acting beneath the indenter, leading to the variation of the nanomechanical response of materials. We observe similar system behavior by comparing different indenter velocities and its impact on created dislocations (see Fig. 3).

The changes of average stress in the system are shown in Figure 4. The inset in Figure 4a shows the time-dependence of the stress occurring in the system. In Figure $4 \mathrm{~b}$ the characteristic stress dependence was presented with the marked regions. In all cases, we observe a complicate evolution of stress changes. With increasing the depth of indentation, we observe only compressive stresses (labelled by region I). Initially, the whole system has residual compressive stresses that evolve as the rate and depth of indentation increases. As the rate of indentation increases, the compressive stress increases linearly, reaching a maximum value for a certain depth of indentation. With an increased the load rate, the
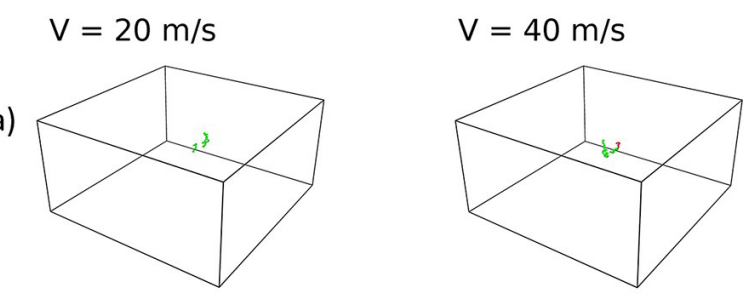

b)
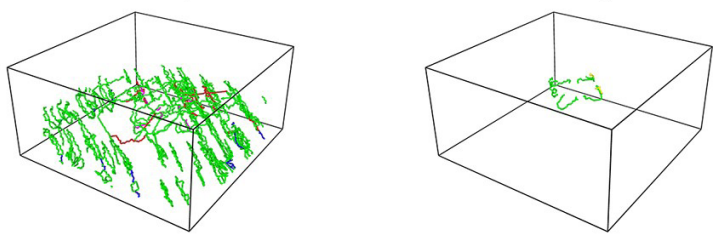

c)
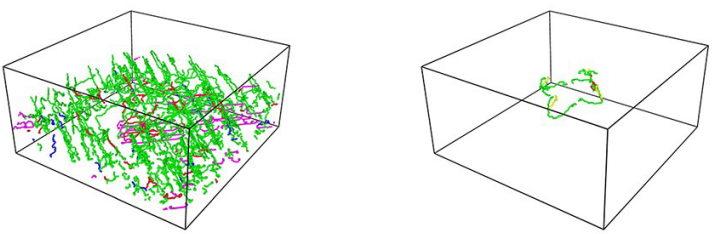

d)
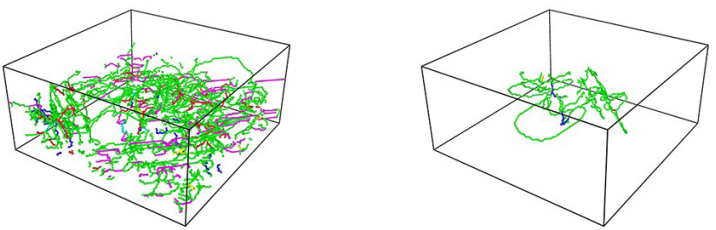

e)
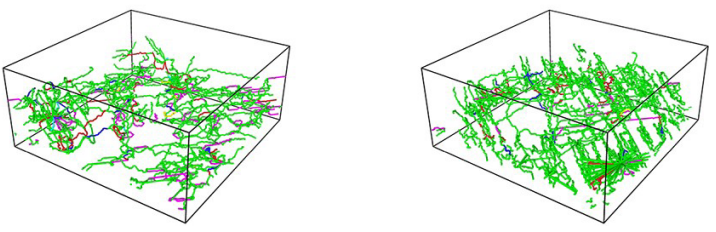

Fig. 3. Snapshots of dislocation evolution during nanoindentation of $\mathrm{Cu}$ for $\mathrm{V}=20 \mathrm{~m} / \mathrm{s}$ at depth (a) $\mathrm{h}=0.4 \mathrm{~nm}$, (b) $\mathrm{h}=0.62 \mathrm{~nm}$, (c) $\mathrm{h}=0.73 \mathrm{~nm}$, (d) $\mathrm{h}=1 \mathrm{~nm}$, (e) $\mathrm{h}=1.4 \mathrm{~nm}$, and for $\mathrm{V}=40 \mathrm{~m} / \mathrm{s}$, respectively. The colors indicate dislocation types obtained from DXA analysis and are marked as follows: green-Shockley, blue-Perfect and red-Stair rod 
value maximum shifts towards a deeper indentation depth and it goes to lower values of stress. The reduction of maximum stress is the result of transferring more kinetic energy into the system by a faster-moving indenter. The supplied energy is used for the nucleation of defects in the system, causing a reduction of stress. This observation is confirmed by the absence of dislocations in the system (see Fig. 5). It can be seen that the response of the system to the operation of the indenter occurs at the same time for different indentation rates (see inset in Fig. 4a). The initial linear change of stress in the compressive direction is associated with the elastic deformation of the crystal lattice of the system. As the indentation rate increases, so does the elastic range. After reaching the maximum, the compressive stress decreases, which suggests the appearance of relaxation mechanisms in the system.

Figure 6 shows that stress distribution during indentation for two rates equals to $20 \mathrm{~m} / \mathrm{s}$ and $40 \mathrm{~m} / \mathrm{s}$ occurring at different indenter depths. For indentation with the lower rate, we observe a regular propagation of stresses in the crystal lattice to a depth of about $0.3 \mathrm{~nm}$. However, for the higher rate, this depth is twice as larger. After crossing the appropriate depths, one can see an irregular distribution of stresses as the result of existence of defects. This result is in a good agreement with the dislocation distribution shown in Figure 3.

After reaching the maximum value, the trend of stress evolution changes in the tensile direction. This region for the clarity of explanation we marked as region II. At a certain indentation depth the stress reaches the minimum stress value. This trend of stress changes is closely connected with the existence of dislocation. Figure 5 shows the dependence the number of dislocation on the indentation rate. For all indentation rates we observe the same dependence of the evolution of the number of dislocations during indentation. At the beginning of the indentation, we do not observe dislocations up to a certain depth. Then, a slow increase in the number of defects and dislocations
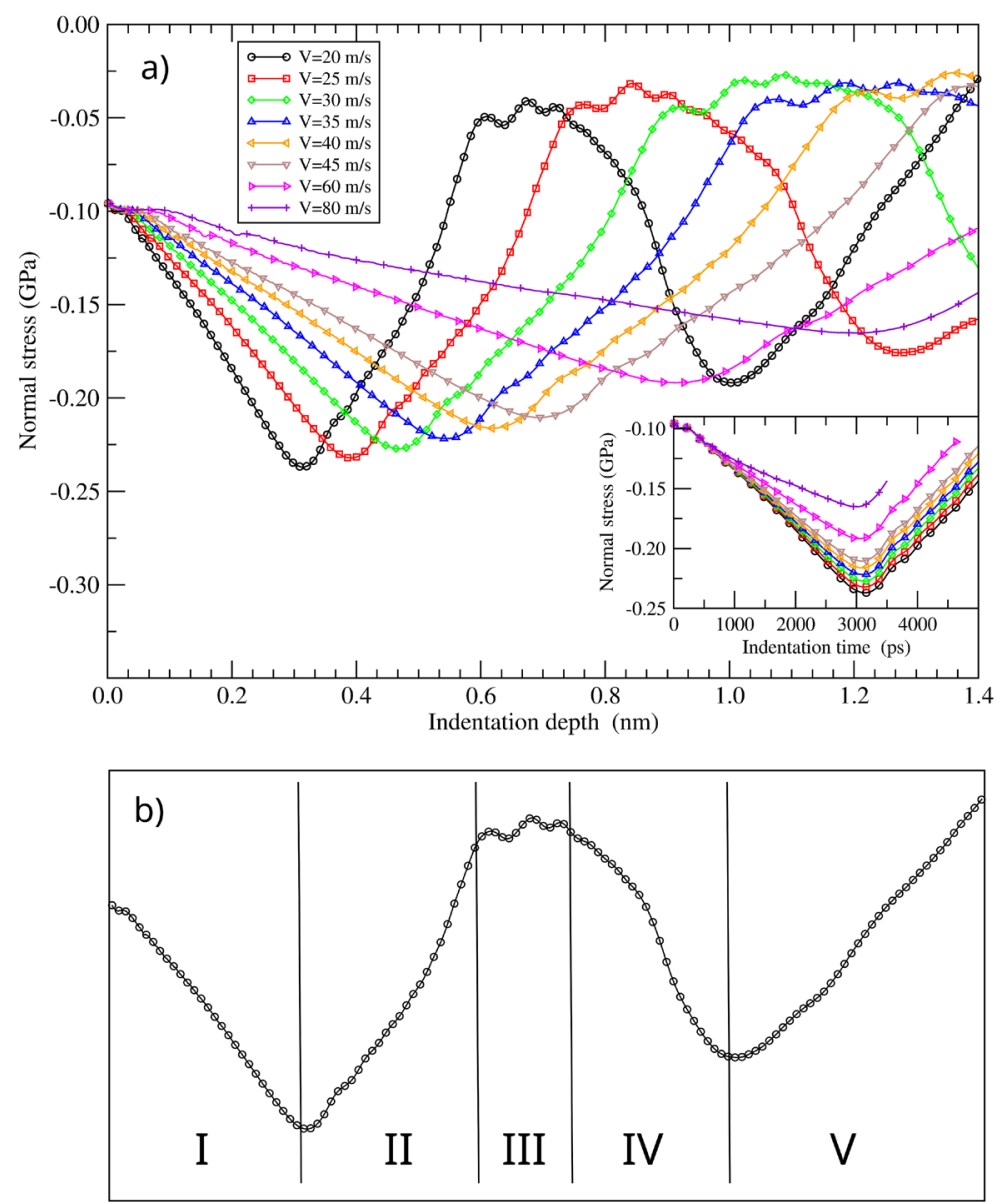

Fig. 4. Normal stress dependence on indentation depth for different rate of indentation. Inset shows the time-dependence of the stress occurring in the system. In part b) of the Figure the regions with different behaviour of stress are marked 


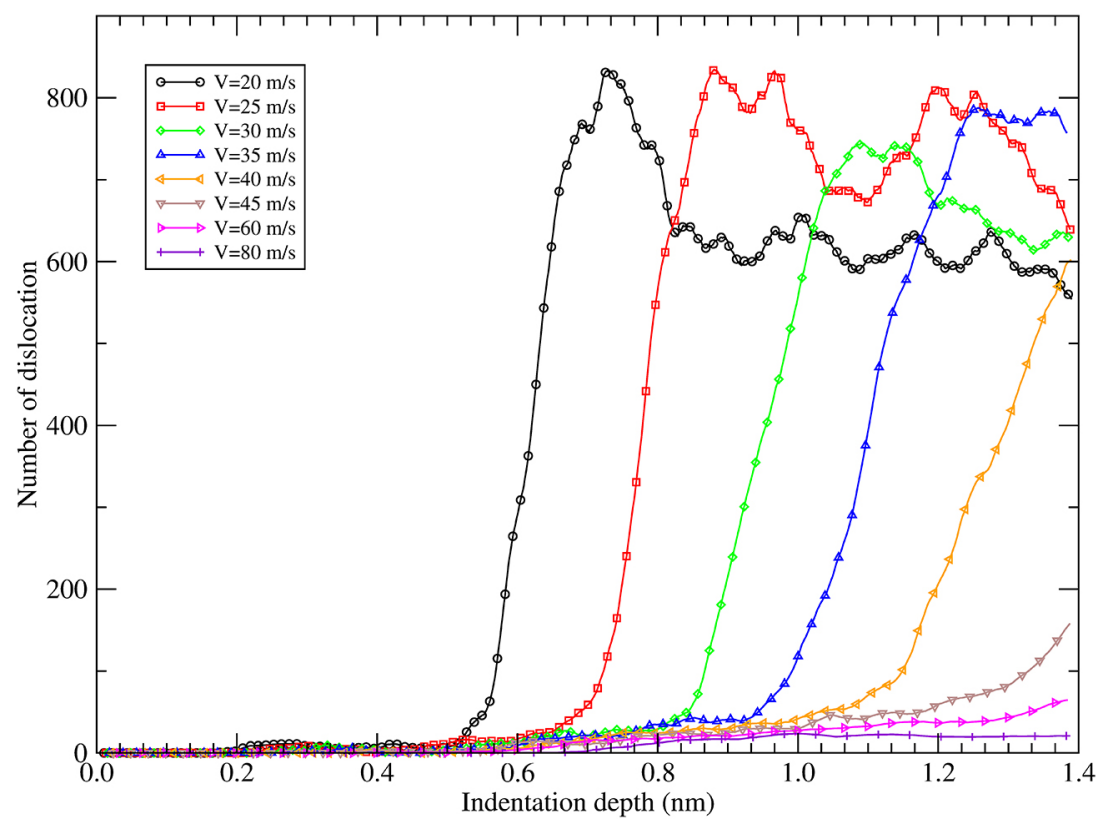

Fig. 5. Evolution of the total number of dislocations during $\mathrm{Cu}$ indentation for different rates of indentation

a)

$$
\mathrm{V}=20 \mathrm{~m} / \mathrm{s}
$$

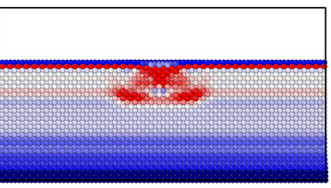

b)

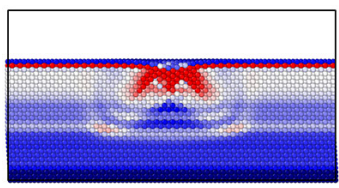

c)

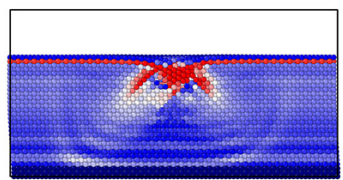

d)

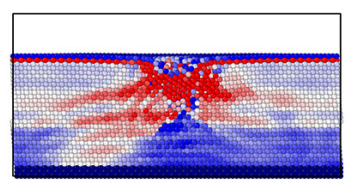

e)

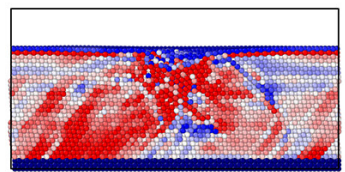

f)

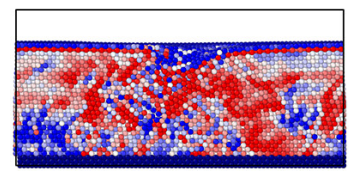

g)

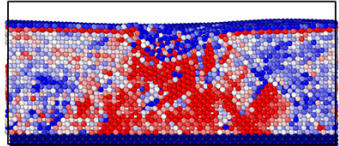

$$
\mathrm{V}=40 \mathrm{~m} / \mathrm{s}
$$
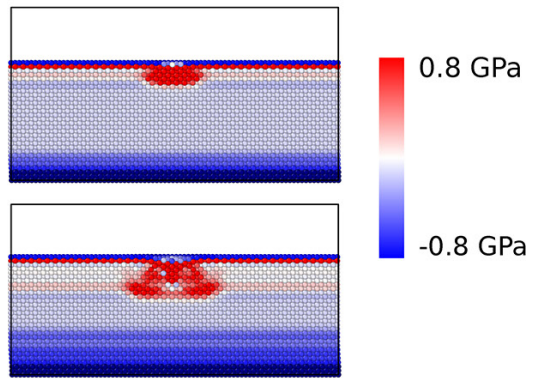

$0.8 \mathrm{GPa}$
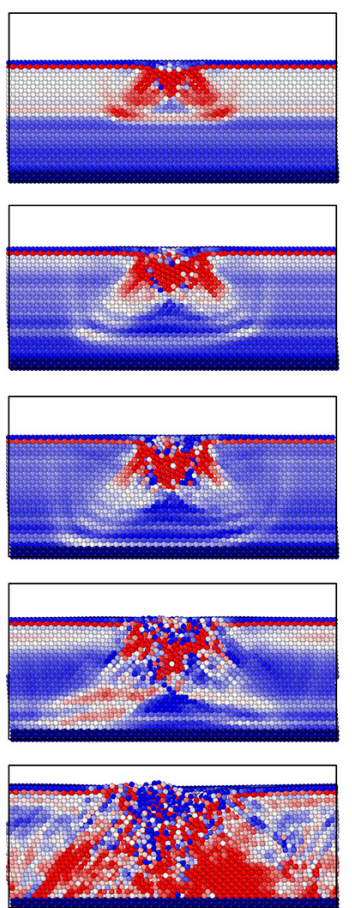

Fig. 6. Snapshots of stress evolution at (a) $h=0.1 \mathrm{~nm}$, (b) $h=0.2 \mathrm{~nm}$, (c) $h=0.3 \mathrm{~nm}$, (d) $h=0.5 \mathrm{~nm}$, (e) $\mathrm{h}=0.6 \mathrm{~nm}$, (f) $\mathrm{h}=0.8 \mathrm{~nm}$, (g) $\mathrm{h}=1.4 \mathrm{~nm}$ depth in the $\mathrm{Z}$ axis for indentation rate equal to $20 \mathrm{~m} / \mathrm{s}$ and $40 \mathrm{~m} / \mathrm{s}$. Stress values were determined according to the colors on the legend 
is observed, followed by a sharp increase in the number of dislocations in the system. A further increase in the depth of indentation causes a decrease in the number of dislocations. The change of stress in the direction of tensile (region II) is associated with the start of the process of creation of dislocations visible as a slight increase in the number of dislocations in Figure 5.

Detailed analysis performed by the DXA method showed the presence of three types of dislocations. We have found that these dislocations are mainly of the Shockley type 1/6 [112] that typically occur in tensile FCC metals. However, we also found Perfect and Stair rod type of dislocation. Figure 3 shows a distribution of dislocations for two rates of indentation: $\mathrm{V}=20 \mathrm{~m} / \mathrm{s}$ and $\mathrm{V}=40 \mathrm{~m} / \mathrm{s}$. The visualizations shown indicate that for higher rates, dislocations are created only in the vicinity of the indenter for deeper depth. However, for lower rates of indentation, dislocations appeared throughout the system for a smaller indenter depth. The results obtained correlate with those presented in Figure 5.

Next, all the curves in Figure 4 reach a minimum value of compressive stress equal to -0.05 (marked as region III). This keeping of stress changes at a similar level is caused on the one hand by a rapid increase in the number of dislocations in this area (see Fig. 5) and by initiating the structure transformation of the FCC area. This transformation is visible in Figure 7 . The increase in the number of dislocations combined with the increase in the number of HCP areas (prismatic loops) is often associated with plastic deformations in the system. A DXA analysis revealed that these dislocations are responsible for the presence of areas with HCP ordering. Figure 7 shows a direct correlation between the decrease in the percentage of the areas of the structure of FCC and the increase in the structure of HCP and BCC. CNA analysis did not show other types of crystallographic structures. This mechanism allows to retain the stress level in the sample almost constant (see Fig. 4).

Looking at Figure 4 (region IV), we observe a renewed increase in compressive stress. The increasing compressive stress mentioned above is a result of the merging of smaller volumes occupied by the HCP structure into larger volumes and the return of a part of volumes to the FCC and BCC structures (see Fig. 7). This phenomenon is an inverse process to the formation of defects occurring in region II, which caused stress relaxation. In this case, it causes an increase in stress.

In region $\mathrm{V}$, we observe changes that are a consequence of those made in the previous region (IV). As the indenter's depth increases, we do not observe a percentage change in the HCP structure. At the same time, we notice an increase of areas with the FCC structure at the expense of disappearance of areas with a the BCC structure. This results in relaxation of compressive stresses in the system.

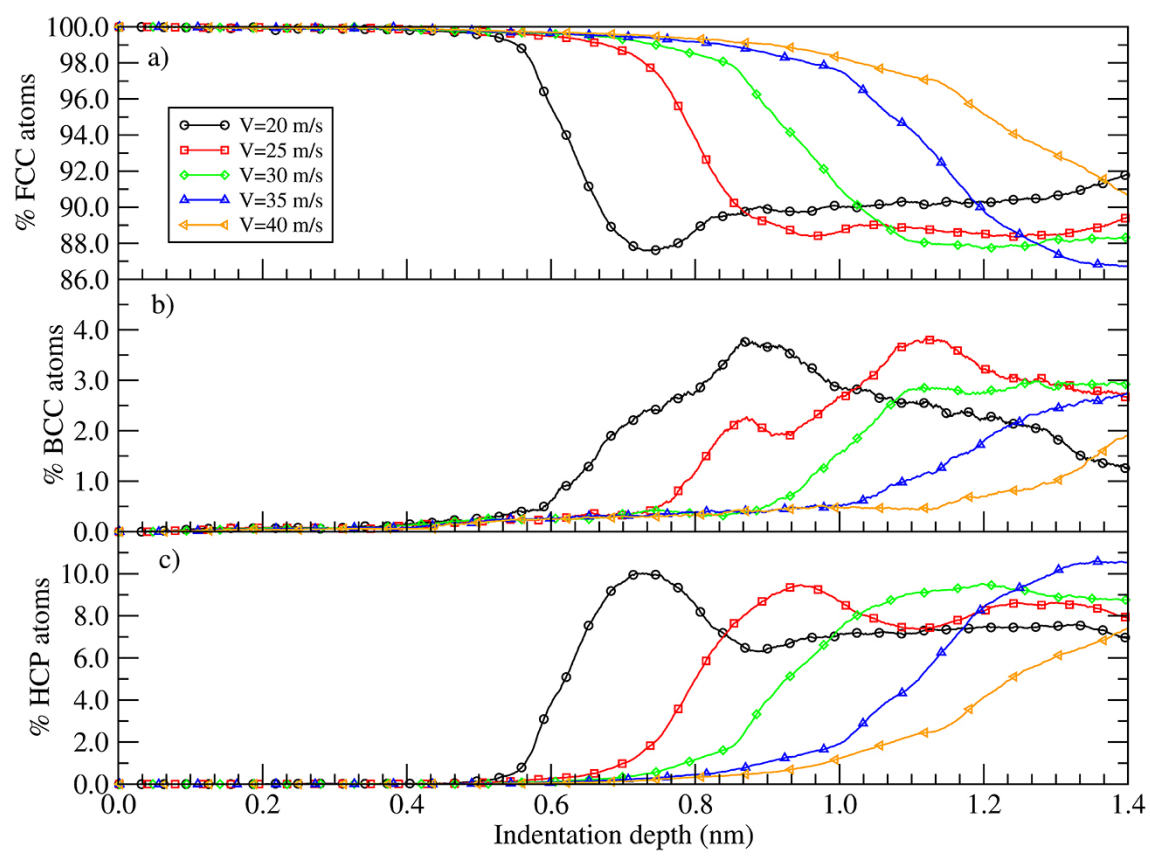

Fig. 7. Variation of percentage of (a) FCC (b) BCC and (c) HCP atoms in the volume of sample as a function of indentation depth for different rates of indentation. The results were obtained from CNA analysis 


\section{CONCLUSIONS}

The study presents the results of MD simulation of the indentation of $\mathrm{Cu}$ films using diamond indenter. The correlation between structure, defects and stress evolution in $\mathrm{Cu}$ systems are obtained. The most interesting results of our calculations include the following:

- The deviation from the linear dependence of the force of indentation depends on the rate of response of the system.

- During indentation, the average total normal stress in the system is only compressive. The initial range of linear dependence of stress evolution also depends on the rate of indentation. After the linear stage, we observe oscillating changes in stress evolution.

- In the range of the linear evolution of stress, dislocations occur only close to the indenter surface.

- The creation of dislocations is responsible for the observed oscillations of stress changes and are directly connected to the structural changes in the system.

- During indentation of the $\mathrm{Cu}$ (001) FCC, the formation of the HCP and BCC structures is observed.

\section{REFERENCES}

1. Kumar A., Ahluwalia P.K. Semiconductor to metal transition in bilayer transition metals dichalcogenides $\mathrm{MX}_{2}(\mathrm{M}=\mathrm{Mo}, \mathrm{W} ; \mathrm{X}=\mathrm{S}, \mathrm{Se}, \mathrm{Te})$. Modelling and Simulation in Materials Science and Engineering. $2013 ; 21(6)$ : 065015.

2. Xie Z., Hui L., Wang J., Zhu G.A., Chen Z., Li C. Electronic and optical properties of monolayer black phosphorus induced by bi-axial strain. Computational Materials Science. 2018; 144: 304-314.

3. Valdes N., Lee J., Shafarman W. Comparison of $\mathrm{Ag}$ and $\mathrm{Ga}$ alloying in low bandgap $\mathrm{CuInSe}_{2}$-based solar cells. Solar Energy Materials and Solar Cells. 2019; 195: 155-159.

4. Chen L., Liu Y., Yang K., Lan P., Cui Y., Luo H., Liu B., Gao Y. Theoretical study of the electronic and optical properties of rare-earth $(\mathrm{RE}=\mathrm{La}, \mathrm{Ce}, \mathrm{Pr}$, $\mathrm{Nd}, \mathrm{Eu}, \mathrm{Gd}, \mathrm{Tb}$ )-doped $\mathrm{VO}_{2}$ nanoparticles. Computational Materials Science. 2019; 161: 415-421.

5. Zhong Y., Xia X., Shi F., Zhan J., Tu J., Fan H.J. Transition metal carbides and nitrides in energy storage and conversion. Advanced Science. 2016; 3(5): 1500286.

6. Gunther E., Mehling H., Hiebler S. Modeling of subcooling and solidification of phase change ma- terials. Modelling and Simulation in Materials Science and Engineering. 2007; 15(8): 879-892.

7. Yan C., Hao L., Hussein A., Young P., Huang J., Zhu W. Microstructure and mechanical properties of aluminium alloy cellular lattice structures manufactured by direct metal laser sintering. Materials Science and Engineering: A. 2015; 628: 238-246.

8. Kiely J.D., Houston J.E. Nanomechanical properties of Au (111), (001), and (110) surfaces. Physical Review B. 1998; 57: 12588-12594.

9. Chocyk D., Zientarski T. Molecular dynamics simulation of $\mathrm{Ni}$ thin films on $\mathrm{Cu}$ and $\mathrm{Au}$ under nanoindentation. Vacuum. 2018; 47: 24-30.

10. Saillard A., Cherkaoui M., El Kadiri H. Stress-induced roughness development during oxide scale growth on a metallic alloy for SOFC interconnects. Modelling and Simulation in Materials Science and Engineering. 2010; 19(1): 015009.

11. Singh M., Sridhara B.K., Shridhar T.N. Studies on nanostructure aluminium thin film coatings deposited using DC magnetron sputtering process. Materials Science and Engineering Conference Series. 2016; 149(1): 012071-012080.

12. Demas N.G., Lorenzo-Martin C., Ajayi O.O., Erck R.A., Shareef I. Measurement of Thin-film Coating Hardness in the Presence of Contamination and Roughness: Implications for Tribology. Metall. Mater. Trans. A. 2016; 47: 1629-1640.

13. DebRoy T., Wei H.L., Zuback J.S., Mukherjee T., Elmer J.W., Milewski J.O., Beese A.M., Zhang W. Additive manufacturing of metallic componentsprocess, structure and properties. Progress in Materials Science. 2018; 92: 112-224.

14. Lewandowski J.J., Seifi M. Metal additive manufacturing: a review of mechanical properties. Annual Review of Materials Research. 2016; 46: 151-186.

15. Liu J., Liang T., Lai W., Liu Y. Morphology evolution and defect distribution in irradiated graphite from molecular dynamics. Computational Materials Science. 2018; 155: 246-255.

16. Deng L., Zhao J., Wang Z. Estimation of residual stress of metal material with yield plateau by continuous spherical indentation method. Materials Research Express. 2020; 7(3): 036537.

17. Valencia F.J., Benjamín Pinto B., Kiwi M., Ruestes C.J., Eduardo M. Bringae E.M., Rogan J. Nanoindentation of polycrystalline Pd hollow nanoparticles: Grain size role. Computational Materials Science. 2020; 179: 109642.

18. Zahabi S., Nouri N., Ziaei-Rad S., Talaei M.S. Effect of iron bicrystal orientation on mechanical properties and dislocation density using molecular dynamics simulations of nanoindentation. Mechanics of Advanced Materials and Structures. 2020. DOI: $10.1080 / 15376494.2020 .1813854$. 
19. Chavoshi S.Z., Xu S. Twinning effects in the single/ nanocrystalline cubic silicon carbide subjected to nanoindentation loading. Materialia. 2018; 3: 304-325.

20. Gerberich W., Nelson J., Lilleodden E., Anderson P., Wyrobek J. Indentation induced dislocation nucleation: the initial yield point. Acta Mater. 1996; 44(9): 3585-3598.

21. Deng L., Liu Q., Wang X., Li J. Load drop and hardness drop during nanoindentation on singlecrystal copper investigated by molecular dynamics. Applied Physics A: Materials Science \& Processing. 2018; 124(11): 743.

22. Zhao K., Mayer A.E., He J., Zhang Z. 2Dislocation based plasticity in the case of nanoindentation. International Journal of Mechanical Sciences. 2018; 148: 158-173.

23. Luu H.T., Dang S.L., Hoang T.V., Gunkelmann N. Molecular dynamics simulation of nanoindentation in $\mathrm{Al}$ and Fe: On the influence of system characteristics. Applied Surface Science. 2021; 551: 149221-149234.

24. Pham V.T., Fang T.H. Interfacial mechanics and shear deformation of indented germanium on silicon (001) using molecular dynamics. Vacuum. 2020; 173: 109184-109196.

25. Li Y., Goyal A., Chernatynskiy A., Jayashankar J.S., Kautzky M.C., Sinnott S.B., Phillpot S.R. Nanoindentation of gold and gold alloys by molecular dynamics simulation. Mater. Sci. Eng. A. 2016; 651: 346-357.

26. Fu T., Peng X., Zhao Y., Feng Ch., Huang Ch., Li Q., Wang Z. MD simulation of effect of crystal orientations and substrate temperature of growth of $\mathrm{Cu} / \mathrm{Ni}$ bilayer films. Applied Physics A: Materials Science \& Processing. 2016; 122: 67-75.

27. Chamani M., Farrahi G.H., Movahhedy M.R. Molecular dynamics simulation of nanoindentation of nanocrystalline $\mathrm{Al} / \mathrm{Ni}$ multilayers. Computational Materials Science. 2016; 112: 175-184.

28. Abdulkadir L.N., Abou-El-Hossein K., Jumare AI., Liman M.M., Olaniyan T.A., Odedeyi P.B. Review of molecular dynamics/experimental study of diamond-silicon behavior in nanoscale machining. The International Journal of Advanced Manufacturing Technology. 2018; 98(1-4): 317-371.

29. Kelchner C.L., Plimpton S.J., Hamilton J.C. Dislocation nucleation and defect structure during surface indentation. Physical Review B. 1998; 58(17): 11085-11088.

30. Li J., Van Vliet K.J., Zhu T., Yip S., Suresh S. Atomistic mechanisms governing elastic limit and incipient plasticity in crystals. Nature. 2002; 418(6895): 307-310.

31. Lilleodden E.T., Zimmerman J.A., Foiles S.M., Nix W.D. Atomistic simulations of elastic deformation and dislocation nucleation during nanoindentation. Journal of the Mechanics and Physics of Solids. 2003; 51(5): 901-920.

32. Zhu T., Li J., Van Vliet K.J., Ogata S., Yip S., Suresh S. Predictive modeling of nanoindentationinduced homogeneous dislocation nucleation in coppe. Journal of the Mechanics and Physics of Solids. 2004; 52(3): 691-724.

33. Van Vliet K.J., Li J., Zhu T., Yip S., Suresh S. Quantifying the early stages of plasticity through nanoscale experiments and simulations. Physical Review B. 2003; 67(10): 104105.

34. Miller R.E., Acharya A. A stress-gradient based criterion for dislocation nucleation in crystals. Journal of the Mechanics and Physics of Solids. 2004; 52(7): 1507-1525.

35. Minor A.M., Lilleodden E.T., Stach E.A., Morris J.W. Direct observations of incipient plasticity during nanoindentation of Al. Journal of Materials Research. 2004; 19(1): 176-182.

36. Minor A.M., Asif S.S., Shan Z., Stach E.A., Cyrankowski E., Wyrobek T.J., Warren O.L. Warren, A new view of the onset of plasticity during the nanoindentation of aluminium. Nat. Mater. 2006; 5(9): 697-702.

37. Schuh C.A., Mason J.K., Lund A.C. Quantitative insight into dislocation nucleation from high-temperature nanoindentation experiments. Nature Materials. 2005; 4(8): 617-621.

38. Mason J.K., Lund A.C., Schuh C.A. Determining the activation energy and volume for the onset of plasticity during nanoindentation. Physical Review B. 2006; 73(5): 054102.

39. Schuh C.A. Nanoindentation studies of materials. Mater. Today. 2006; 9(5): 32-40.

40. Stukowski A., Bulatov V.V., Arsenlis A. Automated identification and indexing of dislocations in crystal interfaces. Modelling and Simulation in Materials Science and Engineering. 2012; 20(8): 085007.

41. Honeycutt J.D., Andersen H.C. Molecular dynamics study of melting and freezing of small LennardJones clusters. Journal of Physical Chemistry. 1987; 91(19): 4950-4963.

42. Zhou X.W., Johnson R.A., Wadley H.N.G. Misfitenergy-increasing dislocations in vapor-deposited CoFe/NiFe multilayers. Physical Review B. 2004; 69: 144113-144123.

43. Chang W., Fang T., Lin S., Huang J. Nanoindentation response of nickel surface using molecular dynamics simulation. Molcular Simulation. 2010; 36: 815-822.

44. Hsieh J., Ju S., Li S., Hwang C. Temperature dependence in nanoindentation of a metal substrate by a diamond like tip. Physical Review B. 2004; 70: 195424 
45. Promyoo R., El-Mounayri H., Varahramyan K. AFM-Based Nanoindentation Process: A Comparative Study. In: ASME 2012 International Manufacturing Science and Engineering Conference, Notre Dame, Indiana USA. June 4-8. 2012; 869-878.

46. Yu Gao Y., Ruestes C.J., Tramontina D.R., Urbassek H.M. Comparative simulation study of the structure of the plastic zone produced by nanoindentation. Journal of the Mechanics and Physics of
Solids. 2015; 75: 58-75.

47. Basinski Z.S., Duesberry M.S., Taylor R. Influence of shear stress on screw dislocations in a model sodium lattice. Can. J. Phys. 1971; 49: 2160-2180.

48. Schuh C.A., Argon A.S., Nieh T.G., Wadsworth J. The transition from localized to homogeneous plasticity during nanoindentation of an amorphous metal. Philosophical Magazine A. 2003; 83(22): 2585-2597. 